makers. Deficiencies in the educational process primarily are in the area of management and organizational requirements, especially in disasters that demand non-traditional approaches to emergency medical services, and as effective decision-makers and representatives in the governmental process. Future disaster medicine leaders will require a broader base of knowledge and experience.

Keywords: disaster medicine; education; emergency medicine; management; organization; specialty

Prehosp Disast Med 2002;17:s28-29.

\section{Introduction of European Master in Disaster Medicine (EMDM)}

\section{F. Della Corte; F. La Mura; M. Debacker; ${ }^{*}$ H. Delooz*}

Chair of Anesthesiology and Intensive Care - Università A. Avogadro, Novara, Italy

"Emeritus Professor - Free University of Brussels, Brussels, Belgium

Introduction: In case of a disaster, the community expects effective relief. Medical involvement is necessary in the planning, in the response, in the coordination, and also in the evaluation of disaster plans, and this requires specific knowledge, the ability to organize an emergency medical system adapted to disaster situation, and the professional skills to provide medical care of high quality even in a hostile environment. Therefore, education and training in all aspects of disaster medicine are essential. The European Master in Disaster Medicine (EMDM: http://dismedmaster.com/home) is a new project that includes theoretical and practical sections together with the use of the new electronic educational tools, organized by the Universities of Novara (Italy) and the Free University of Brussels (Belgium) hosted by the European Center for Disaster Medicine (CEMEC).

Objective: The EMDM aims at training the medically educated professional to respond better in the aftermath of an event that causes a disaster using an innovative educational approach, implementing adult learning (self-study, frontal lessons, practical exercises, etc.). Using distancelearning techniques. At the end of the course, the participants are expected to be able to evaluate risks, participate in planning for disaster preparedness, direct the medical response team in case of disasters, organize and manage evaluation and debriefing sessions, and provide introduction to disaster management for medical response teams.

Methods: The duration of the Master is of one academic year divided into four month segments of self study including the first draft of the dissertation or the research project, two consecutive weeks of an interactive, live-in course concluded by the multiple choice questions and oral evaluation, four months for the redaction of the final version of the research project, and preparation for the interactive Internet assessment. In our vision of distance learning education, effort and care have been taken for implementing an electronic book (part of our "virtual classroom") and a computer/Internet-based, simulated approach to emergency and disaster situations. The electronic book is divided into modules and chapters, which can be uploaded/updated by the authors with simple actions taken in the "administrative" side of the website. The EMDM website assigns a pre-defined level of privilege to every user so that a higher level corresponds to a wider range of actions (authoring tools, forum and polls, administration, etc.). The last version of the authoring tool is a powerful, Java-based WYSIWYG courseware-developing environment, specifically tailored for EMDM didactic needs. The simulation exercises (based on the ITLS system by E Semble) include assessment and resuscitation/stabilization issues, triage (conventional and mass casualty), treatment, tactical evacuation, and casualty management. The casualties are chosen by the teachers using a victim database with over 1,500 records, and can be put in a scenario builder offering multiuser and real time simulation capabilities.

Conclusion: The results and positive feedbacks that EMDM has received during its first Academic Year greatly contributed to help to ameliorate and expand both the technical side and some didactic/organizational issues.

Keywords: Disaster Medicine; distance learning; education; evaluation; planning; simulation

Prebosp Disast Med 2002;17:s29.

\section{Education and Training in Disaster Medicine Azhar Abdul Aziz}

Department of Emergency Medicine, National University of Malaysia Hospital, Kuala Lumpur, Malaysia

Given the global rate of urbanization and industrialization, disasters, though occurring infrequently, are expected to increase quantitatively and worsen qualitatively. Knowledge regarding each event not only is limited, but varies between different disasters. Therefore, education and training are vital in gaining the maximum achievements of mitigation, planning, response, and recovery at any one tragedy.

Personnel from a wide spectrum of agencies must be educated and trained, including those from medical, rescue, military, government administration, NGOs, and all others who are directly and indirectly involved in their management. This programme must cover: 1) Concepts and definitions; 2) Types and classifications; 3) Effects and complications; 4) Phases of disaster cycle; and 5) All other related topics.

It is also important to involve lay public, so that disseminated information and skills may lead to common understanding and implementation of standardized plans. Either these must be conducted at local or regional levels, education and training may employ various techniques, namely: 1) Lectures; 2) Discussions; 3) Case studies; 4) Lab workshops; 5) Table top exercises; 6) Mock drills; and 7) Post mortems.

Other initiatives also should complement the above, such as research studies and organizing seminars and conferences. Evidently, lessons learnt and acquired through this process have, and will continue to improve the management performance and outcome of any disaster event. Keywords: courses; curriculum; disaster medicine; education; plans; training

Prebosp Disast Med 2002;17:s29. 